Journal of New Results in Science
https://dergipark.org.tr/en/pub/jnrs
Research Article
JNRS

\title{
Investigation of the Lorenz number and the carrier concentration of the GaAs semiconductor depending on temperature
}

\author{
Ebru Çopuroğlu 1 (1)
}

\author{
Keywords: \\ Hall Coefficient, \\ Fermi integral, \\ Carrier concentration, \\ Semiconductors
}

\begin{abstract}
As is known, semiconductors are insulators under normal conditions but can become conductive with external excitation. Considering the effects of acting on these materials, the number of free electrons and the electrical conductivity will increase with increasing temperature. The increase in the concentration of free electrons in the semiconductor can be shown as the increase in electrical conductivity. If a semiconductor is exposed to an electric field with increasing concentration, we can have an idea about how the number of free electrons or the speed of free electrons will be affected. It is well known that it is necessary to calculate twoparameter Fermi functions to solve the properties of kinetic effects and electron transport phenomena in semiconductors. Effective methods have been developed for the calculation of two-parameter Fermi functions in literature. In this study, analytical calculations for the Lorenz number and the carrier concentration of the GaAs semiconductor were made using the twoparameter Fermi function.
\end{abstract}

Subject Classification (2020):

\section{Introduction}

Thermoelectric effects observed in various materials, such as metals, semiconductors, and superconductors, occur when a conductor with free temperature carriers is exposed to an electric field under temperature change. The analysis of thermoelectric effects is widely used to study the physical properties of materials [1-12]. Due the fact that developments in determining thermal and electrical properties in solid state materials have a significant role in industrial revolution, there has been an increase in the study of thermoelectric effects in materials. The most known thermoelectric effects are the Joule, Seebeck, Thomson, and Peltier effects in literature. Also, the Lorenz number is the coefficient which determines the thermal conductivity with respect to Wiedemann- Franz Law. It is well known that depending on temperature the Lorenz number deviate significantly from its limit value especially in non-degenerated semiconductors [13-17]. In the 1960s, many studies were carried out to examine the thermoelectric and thermomagnetic properties of semiconductors, to understand the variation of the Lorenz number and carrier concentration with temperature, the type of energy spectrum, the properties of the band structure and their effects on the scattering character. The use of thermoelectric

1ebrucopuroglu@gmail.com (Corresponding Author)

1Department of Physics, Faculty of Arts and Sciences, Tokat Gaziosmanpaşa University, Tokat, Turkey

Article History: Received: 22 Oct 2021 - Accepted: 07 Dec 2021 — Published: 31 Dec 2021 
coefficients to determine the changes in Lorenz number and carrier concentration is essential in this study.

There is a group of semiconductors in which the conduction band has spherical symmetry but cannot be accurately described by known dispersion laws. Therefore, scientists have been interested in deriving formulas for kinetic coefficients under the most general assumptions about the shape of the region and scattering mechanisms. As a result of these studies, the formulas of the fundamental kinetic coefficients are derived with the help of a two-parameter Fermi function by B.M. Askerov [10] for the first time. In this study, an alternative analytical method for evaluating Lorenz number and carrier concentration is suggested by using the two-parameter Fermi function. As an application of the given approach, the calculations have been performed for the GaAs semiconductor.

\section{Material Method}

\subsection{Analytical Investigation of Two-Parameter Fermi Function}

Fermi distribution functions with one-, two- and three-parameters can be used to define the kinetic coefficients of the semiconductor [10-13]. However, it is necessary to use two-parameter Fermi integrals for solutions involving both thermal and electric effects.

Accurate analytical formulas of the two-parameter Fermi functions are of great importance in evaluating problems related to solid state physics, especially kinetic effects and the theory of transport phenomena in semiconductors [12]. The physical properties of carriers and the relationships of semiconductors in non-parabolic energy bands are usually reduced to solving the two-parameter Fermi distribution function for any degree of degeneration. Therefore, the correct analysis and interpretation of this function are important issues that need to be studied. Both numerical and analytical solutions are available for solving the Fermi function. The two-parameter Fermi integral can be defined as [12]:

$$
I_{n, k}^{m}(\eta, \beta)=\int_{0}^{\infty}\left(-\frac{\partial f_{0}(x, \eta)}{\partial x}\right) \frac{x^{m}\left(x+\beta x^{2}\right)^{n}}{(1+2 \beta x)^{k}} d x
$$

Here, $\eta=\frac{\xi}{k_{0} T}$ is the reduced chemical potential, $\beta=\frac{k_{0} T}{\varepsilon_{g}}$ is the amount of deviation from the parabolic, $\varepsilon_{g}$ is forbidden energy bandgap, $\xi$ is the chemical potential, $k_{0}$ is the Boltzman temperature and $f_{0}(x, \eta)$ is the Fermi distribution function defined as follows:

$$
f_{0}(x, \eta)=\frac{1}{1+e^{x-\eta}}
$$

Effective methods have been developed for the calculation of two-parameter Fermi functions. Zawadzki et al. [18] proposed a general procedure for calculating two-parameter Fermi functions for values of integral parameters. Here, $-5 \leq \eta \leq 20$ and $0 \leq \beta \leq 1$. Askerov used the numerical method to approximate two-parameter Fermi functions. This proposed approach is correct in the region of $\eta \geq 10$ strongly degenerate and is written as $[11,12]$ :

$$
\begin{aligned}
I_{n, k}^{m}(\eta, \beta) \approx \frac{\eta^{n+m}(1+\beta \eta)^{n}}{(1+2 \beta \eta)^{k}}\left\{1+\frac{\pi^{2}}{6}\left[\frac{(n+m)(n+m-1)}{\eta^{2}}+\frac{2 n(n+m) \beta}{\eta(1+\beta \eta)}-\frac{4 k(n+m) \beta}{\eta(1+2 \beta \eta)}\right.\right. \\
\left.\left.+\frac{n(n-1) \beta^{2}}{(1+\beta \eta)^{2}}-\frac{4 n k \beta^{2}}{(1+2 \beta \eta)(1+\beta \eta)}+\frac{4 k(k+1) \beta^{2}}{(1+2 \beta \eta)^{2}}\right]\right\} \text { for }\left(\frac{k_{0} T}{\zeta}\right)<<1
\end{aligned}
$$


In the non-degenerate case, the two-parameter Fermi functions can be evaluated by using the following given formula:

$$
I_{n, k}^{m}(\eta, \beta) \approx e^{\eta} \Gamma(n+m+1) \frac{(1+n \beta+m \beta)^{n}}{(1+2 n \beta+2 m \beta)^{k}} \text { for } \eta \leq-5 \text { and } n \neq 0 \text { or } m \neq 0
$$

Here $\Gamma(\alpha)$ is the well-known Gamma function. Zawadzki examined different methods used in the evaluation of two-parameter Fermi functions [18]:

$$
I_{n, k}^{m}(\eta, \beta)=\sum_{p=0}^{\infty} a_{p} \beta^{p} F_{n+m+p-1}(\eta)
$$

and

$$
I_{n, k}^{m}(\eta, \beta)=\sum_{p=0}^{\infty} \sum_{r=1}^{\infty}(-1)^{r-1} a_{p} \beta^{p} \frac{e^{r \eta}}{r^{n+m}} \text { for }<1
$$

Here,

$$
a_{p}=(n+m+p) ! \sum_{l=0}^{p} \frac{[n(n-1) \ldots(n-p+l+1)][-k(-k-1) \ldots(-k-p-l+1)] 2_{l}}{(p-l) ! l !}
$$

$F_{p}(\eta)$ is the Fermi function and defined as:

$$
F_{p}(\eta)=\int_{0}^{\infty} \frac{e^{x-\eta_{x} p}}{e^{x-\eta}+1} d x
$$

The Fermi functions are well-known and can be studied with standard techniques without numerical difference in its use [19-21]. One of the efficient methods is the using following binomial expansion method for the evaluation of Fermi functions [22]:

$$
(x \pm y)^{n}= \begin{cases}\sum_{m=0}^{\infty}( \pm 1)^{m} f_{m}(n) x^{n-m} y^{m} \text { for noninteger } n \\ \sum_{m=0}^{n}( \pm 1)^{m} f_{m}(n) x^{n-m} y^{m} \text { for integer } n\end{cases}
$$

Here $f_{m}(n)$ is the binomial function:

$$
f_{m}(n)= \begin{cases}\frac{n(n-1) \ldots(n-m+1)}{m !} & \text { for integer } n \\ \frac{(-1)^{m} \Gamma(m-n)}{m ! \Gamma(-n)} & \text { for noninteger } n\end{cases}
$$

By considering equation (2.9), the series expansion formulas for two-parameter Fermi functions were obtained following as [21]:

for $n \neq 0, k \neq 0, m \neq 0$ 


$$
\begin{aligned}
& I_{n k}^{m}(\eta, \beta) \\
& =e^{-\eta} \lim _{N \rightarrow \infty} \sum_{i=0}^{N} f_{i}(-k) \begin{cases}\sum_{j=0}^{n} f_{j}(n)\left[\beta^{i+j} 2^{i} P_{m+n+i+j}\left(\eta, \frac{1}{2 \beta}\right)\right. & \\
\left.+\beta^{j-k-i} 2^{-k-i} Q_{m+n-k-i+j}\left(\eta, \frac{1}{2 \beta}\right)\right] & \text { for integer } n \\
\lim _{N \rightarrow \infty} \sum_{j=0}^{N} f_{j}(n)\left[\beta^{i+j} 2^{i} P_{m+n+i+j}\left(\eta, \frac{1}{2 \beta}\right)\right. & \\
\left.+\beta^{n-j-k-i} 2^{-k-i} Q_{m+2 n-k-i+j}\left(\eta, \frac{1}{2 \beta}\right)\right] & \text { for noninteger } n\end{cases}
\end{aligned}
$$

for $n=0, k=0$ and $m \neq 0$

$$
I_{00}^{m}(\eta, \beta)=e^{-\eta}\left\{\begin{array}{l}
\lim _{N \rightarrow \infty} \sum_{i=0}^{N} \frac{f_{i}(-2)}{(1+i)^{m+1}}\left[e^{i \eta}(-1)^{m+1} \gamma(m+1,-(1+i) \eta)\right. \\
\left.+e^{\eta(2+i)} \Gamma(m+1,(1+i) \eta)\right] \text { for } \eta>0 \\
\lim _{N \rightarrow \infty} \sum_{i=0}^{N} f_{i}(-2) \frac{e^{\eta(2+i)} \Gamma(m+1)}{(1+i)^{m+1}} \text { for }<0
\end{array}\right.
$$

for $n \neq 0, k=0, m=0$ and $\eta>0$

$$
I_{n 0}^{0}(\eta, \beta)=e^{-\eta}\left\{\begin{array}{l}
\sum_{i=0}^{n} f_{i}(n) \beta^{i} L_{n+i}(\eta) \quad \text { for integer } n \\
\lim _{N \rightarrow \infty} \sum_{i=0}^{N} f_{i}(n)\left(\beta^{i} P_{n+i}\left(\eta, \frac{1}{\beta}\right)+\beta^{n-i} Q_{2 n-i}\left(\eta, \frac{1}{\beta}\right)\right) \quad \text { for noninteger } n
\end{array}\right.
$$

for $n \neq 0, k=0, m=0$ and $\eta<0$

$$
I_{n 0}^{0}(\eta, \beta)=e^{-\eta}\left\{\begin{array}{l}
\sum_{i=0}^{n} f_{i}(n) \beta^{i} \lim _{N \rightarrow \infty} \sum_{j=0}^{N} f_{j}(-2) \frac{e^{(2+j) \eta} \Gamma(n+i+1)}{(1+j)^{n+i+1}} \text { forninteger } \\
\lim _{N \rightarrow \infty} \sum_{i=0}^{N} f_{i}(n) \sum_{j=0}^{N} f_{j}(-2) e^{(2+j) \eta}\left[\frac{\beta^{i} \gamma(n+i+1,(1+j) / \beta)}{(1+j)^{n+i+1}}\right. \\
\left.+\frac{\beta^{n-i} \Gamma(2 n-i+1,(1+j) / \beta)}{(1+j)^{2 n-i+1}}\right] \text { fornnoninteger }
\end{array}\right.
$$

for $n=0, k<0, m=0$ 


$$
I_{0 k}^{0}(\eta, \beta)=e^{-\eta}\left\{\begin{array}{lc}
\sum_{i=0}^{k} f_{i}(k)(2 \beta)^{i} \lim _{N \rightarrow \infty} \sum_{j=0}^{N} f_{j}(-2)\left(\frac{e^{-i \eta}(-1)^{i+1} \gamma(i+1,-(1+j) \eta)}{(1+j)^{i+1}}\right. & \text { for } \eta>0 \\
\left.+\frac{\left.e^{(2+j) \eta} \Gamma(i+1,(1+j) \eta)\right)}{(1+j)^{i+1}}\right) & \text { for } \eta<0 \\
\sum_{i=0}^{k} f_{i}(k)(2 \beta)^{i} \lim _{N \rightarrow \infty} \sum_{j=0}^{N} f_{j}(-2) \frac{e^{(2+i) \eta} \Gamma(i+1)}{(1+j)^{i+1}} &
\end{array}\right.
$$

for $n \neq 0, k<0, m=0$

$$
I_{n k}^{0}(\eta, \beta)=e^{-\eta} \begin{cases}\sum_{i=0}^{n} f_{i}(n) \beta^{i} \sum_{j=0}^{k} f_{j}(k)(2 \beta)^{j} \lim _{N \rightarrow \infty} \sum_{l=0}^{N} f_{l}(-2)\left(\frac{e^{-l \eta}(-1)^{n+i+j+1}}{(1+l)^{n+i+j+1}}\right. & \\ \left.\times \gamma(n+i+j+1,-(1+l) \eta)+\frac{\left.e^{(2+l) \eta} \Gamma(n+i+j+1,(1+l) \eta)\right)}{(1+l)^{n+i+j+1}}\right) & \text { for } \eta>0 \\ \sum_{i=0}^{n} f_{i}(n) \beta^{i} \sum_{j=0}^{k} f_{j}(k)(2 \beta)^{j} \lim _{N \rightarrow \infty} \sum_{l=0}^{N} f_{l}(-2) \frac{e^{(2+l) \eta} \Gamma(n+i+j+1)}{(1+l)^{n+i+j+1}} & \text { for } \eta<0\end{cases}
$$

The auxiliary functions $P_{n}(p, q), Q_{n}(p, q)$ and $L_{n}(p)$ occurring in Eqs. (2.11) to (2.16) are expressed through the special functions and can be written as, respectively:

for $p>0$

$$
\begin{gathered}
P_{n}(p, q)=\lim _{N \rightarrow \infty} \sum_{i=0}^{N} f_{i}(-2)\left\{\begin{array}{lc}
\left(\frac{(-1)^{n+1} e^{-i p} \gamma(n+1,-(1+i) p)}{(1+i)^{n+1}}+e^{(2+i) p}\left[p^{n+1} E_{-n}((1+i) p)\right.\right. \\
\frac{\left.\left.-q^{n+1} E_{-n}((1+i) q)\right]\right)}{(-1)^{n+1} e^{-i p} \gamma(n+1,-(1+i) q)} & \text { for } p \leq q \\
(1+i)^{n+1} & \text { for } p>q
\end{array}\right. \\
Q_{n}(p, q)=\lim _{N \rightarrow \infty} \sum_{i=0}^{N} f_{i}(-2)\left\{\begin{array}{lc}
\left(\frac{e^{(2+i) p} \Gamma(n+1,(1+i) p)}{(1+i)^{n+1}}+e^{-i p}\left[q^{n+1} E_{-n}(-(1+i) q)\right.\right. \\
\left.\left.-p^{n+1} E_{-n}(-(1+i) p)\right]\right) \\
\frac{e^{(2+i) p} \Gamma(n+1,-(1+i) q)}{(1+i)^{n+1}} & \text { for } p>q
\end{array}\right. \\
\text { for } p \leq q
\end{gathered}
$$

for $p<0$

$$
\begin{aligned}
& P_{n}(p, q)=\lim _{N \rightarrow \infty} \sum_{i=0}^{N} f_{i}(-2) \frac{e^{(2+i) p} \gamma(n+1,(1+i) q)}{(1+i)^{n+1}} \\
& Q_{n}(p, q)=\lim _{N \rightarrow \infty} \sum_{i=0}^{N} f_{i}(-2) \frac{e^{(2+i) p} \Gamma(n+1,(1+i) q)}{(1+i)^{n+1}}
\end{aligned}
$$




$$
L_{n}(p)=\lim _{N \rightarrow \infty} \sum_{i=0}^{N} f_{i}(-2)\left(\frac{(-1)^{n+1} e^{-i p} \gamma(n+1,-(1+i) p)}{(1+i)^{n+1}}+\frac{e^{(2+i) p} \Gamma(n+1,(1+i) p)}{(1+i)^{n+1}}\right)
$$

where $\Gamma(\alpha)$ and $\Gamma(\alpha, x)$ are the incomplete Gamma functions defined by [22]

$$
\Gamma(\alpha)=\int_{0}^{\infty} t^{\alpha-1} e^{-t} d t
$$

and

$$
\begin{aligned}
& \Gamma(\alpha, x)=\int_{x}^{\infty} t^{\alpha-1} e^{-t} d t \\
& \gamma(\alpha, x)=\int_{0}^{x} t^{\alpha-1} e^{-t} d t
\end{aligned}
$$

In this study, the suggested formulas (2.11-2.16) for the two-parameter Fermi function has been used in Lorenz number and carrier concentration calculations.

\subsection{The Definition of Lorenz Number and Carrier Concentration of a Semiconductor}

As is known, the carrier concentration in the conduction band is shown as follows [10-12]:

$$
\begin{gathered}
n=\int_{E_{c}}^{E_{w p}} f(E) g(E) d E \\
n=\int_{E_{c}}^{E_{w p}} \frac{\left(4 \pi 2 m_{e}^{*} / h^{2}\right)^{\frac{3}{2}}\left(E-E_{c}\right)^{1 / 2}}{1+e^{\frac{E-E_{F}}{k T}}}
\end{gathered}
$$

By the use of two-parameter Fermi function $I_{n k}^{m}(\eta, \beta)$, the carrier concentration can be defined as [23]:

$$
n=\frac{\left(2 m_{n} k_{B} T\right)^{\frac{3}{2}}}{3 \pi^{2} \hbar^{3}} I_{\frac{3}{2}, 0}^{0}(\eta, \beta)
$$

As we know, the Wiedeman-Franz law and its relation with the Lorenz number in the general form are as follows:

$$
\frac{\kappa}{\sigma}=A(r, \eta, \beta)\left(\frac{k_{B}}{e}\right)^{2} T=L(r, \eta, \beta) T
$$

where $L(r, \eta, \beta)$ is Lorenz number, $\kappa$ is thermal conductivity, $\sigma$ is electric conductivity, $k_{B}$ is Boltzmann constant, $e$ is the electron charge, $T$ is temperature. Also, $A(r, \eta, \beta)$ function is defined as:

$$
A(r, \eta, \beta)=\frac{I_{r+1,2}^{2}(\eta, \beta)}{I_{r+1,2}^{0}(\eta, \beta)}+\left(\frac{I_{r+1,2}^{1}(\eta, \beta)}{I_{r+1,2}^{0}(\eta, \beta)}\right)^{2}
$$


It is clear that the Lorenz number can be written as [23]:

$$
L(r, \eta, \beta)=A(r, \eta, \beta)\left(\frac{k_{B}}{e}\right)^{2}
$$

Thus, the Lorenz number turns into the following form by using $I_{n k}^{m}(\eta, \beta)$ two-parameter Fermi functions:

$$
L(r, \eta, \beta)=\left(\frac{I_{r+1,2}^{2}(\eta, \beta)}{I_{r+1,2}^{0}(\eta, \beta)}+\left(\frac{I_{r+1,2}^{1}(\eta, \beta)}{I_{r+1,2}^{0}(\eta, \beta)}\right)^{2}\right)\left(\frac{k_{B}}{e}\right)^{2} .
$$

As can be seen from formulas (2.27) and (2.31), the carrier concentration and the Lorenz number of a semiconductor can be defined using two-parameter Fermi functions. To show the efficiency of given algorithms, the Lorenz number and carrier concentration calculations of GaAs semiconductors have been done for wide temperature ranges (Figure 1 and 2). In addition, the comparative results of the carrier concentration with the available semi-empirical study [24] are presented in Table 1.

Table 1. Temperature dependence of carrier concentration of GaAs semiconductor (in semi-empirical methods [24] carrier concentration is taken as $\left.n=1.83 \times 10^{21} T^{3 / 2}\right)(N=150)$

\begin{tabular}{|c|c|c|}
\hline $\boldsymbol{T}$ & Ref. [24] & This study \\
\hline 150 & $3.361 \mathrm{E}+24$ & $2.981 \mathrm{E}+24$ \\
\hline 100 & $1.84 \mathrm{E}+24$ & $1.162 \mathrm{E}+24$ \\
\hline 80 & $1.309 \mathrm{E}+24$ & $0.958 \mathrm{E}+24$ \\
\hline 50 & $6.47 \mathrm{E}+23$ & $5.651 \mathrm{E}+23$ \\
\hline 40 & $4.629 \mathrm{E}+23$ & $3.487 \mathrm{E}+23$ \\
\hline
\end{tabular}

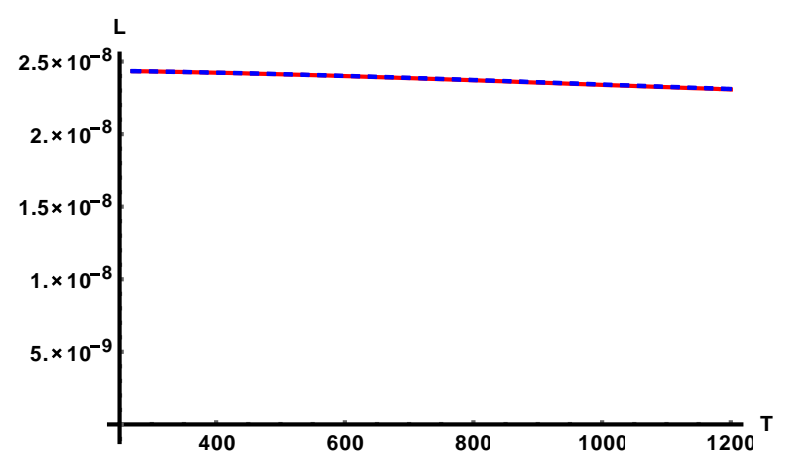

Figure 1. Temperature dependence of Lorenz number of GaAs semiconductor $(N=150)$ (red lineanalytical results from suggested formula in this study, blue line-Mathematica numerical results)

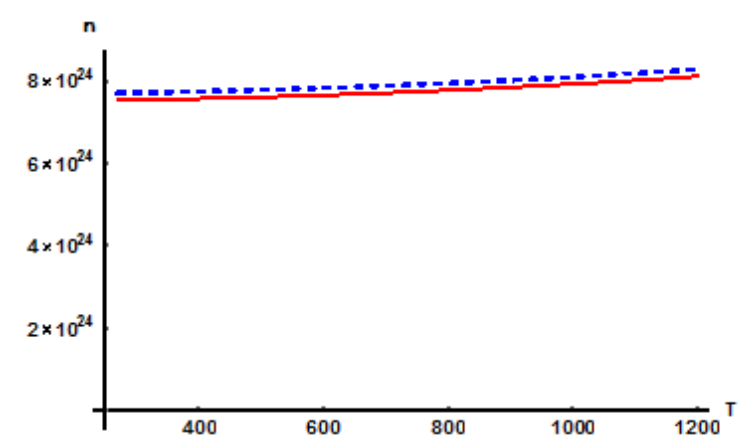

Figure 2. Temperature dependence of carrier concentration of GaAs semiconductor $(N=150)$ (red line-analytical results from suggested formula in this study, blue line-Mathematica numerical results) 


\section{Conclusion}

The thermoelectric effect is based on examining the thermal effect acting on the semiconductor. For this purpose, in this study, the effects of the thermal effect on basic parameters of a semiconductor are numerically discussed. The carrier concentration variation, which is the main variable of the thermal conductivity relation that can be solved by the two-parameter Fermi function, has been investigated for GaAs semiconductors with increasing temperature. In order to demonstrate the effectiveness of our study, the calculation results obtained from available semi-empirical methods for the carrier concentration of GaAs semiconductors have been presented in Table 1. In Fig.1 and 2, the comparisons of the Lorenz number and carrier concentration calculation results of the GaAs semiconductor with the Mathematica numerical method have been demonstrated concerning temperature. Our calculation results are in good agreement with those obtained from numerical and semi-empirical methods. In all calculations the upper limits of summation $(N)$ have been taken as 150. Also, in our previous paper [21] one can see the convergence of derived expression for $I_{n k}^{m}(\eta, \beta)$ as a function of summation limits $\mathrm{N}$. It is well known that the little discrepancy of comparisons in Table 1 is due to the fact that the semi-empirical method neglects some parameters in calculations. Our analytical method has no restriction in its use and can apply to other semiconductors.

\section{Author Contributions}

All authors contributed equally to this work. The author read and approved the last version of the manuscript.

\section{Conflicts of Interest}

The author declares no conflict of interest.

\section{References}

[1] C. Kittel, Introduction to solid state physics, John Wiley \& Sons. Inc., Sixth Edition, New York, 1986.

[2] N. W. Ashcroft, N.D. Mermin, Solid state physics, Philadelphia Pa., New York, 1976.

[3] H. P. Myers, Introductory solid state physics. CRC Press, London, 1997.

[4] E. O. Kane, Band structure of indium antimonide, Journal of Physics and Chemistry of Solids, 1(4), (1957) 249-261.

[5] B. Šantić, B., U. V. Desnica, Thermoelectric effect spectroscopy of deep levels-application to semiinsulating GaAs, Applied Physics Letters, 56(26), (1990) 2636-2638.

[6] L. Zhu, R. Ma, L. Sheng, M. Liu, D. N. Sheng, Universal thermoelectric effect of Dirac fermions in graphene, Physical Review Letters, 104(7), (2010) 076804.

[7] D. Segal, Thermoelectric effect in molecular junctions: A tool for revealing transport mechanisms, Physical Review B, 72(16), (2005) 165426.

[8] P. E. Nielsen, P. L. Taylor, Theory of thermoelectric effects in metals and alloys, Physical Review B, 10(10), (1974) 4061.

[9] G. Sansone, A. Ferretti, L. Maschio, Ab initio electronic transport and thermoelectric properties of solids from full and range-separated hybrid functionals, The Journal of Chemical Physics, 147(11), (2017) 114101. 
[10] B. M. Askerov, F. M. Gashimzade, Determination of parameters of degenerate semiconductors, Physica Status Solidi (b), 21(2), (1967) 155-158.

[11] B. M. Askerov, Kinetic effects in semiconductors, Science, Leningrad, 1970.

[12] B. M. Askerov, Electron transport phenomena in semiconductors. World Scientific, London, 1994.

[13] H. S. Kim, Z. M. Gibbs, Y. Tang, H. Wang, G. J. Snyder, Characterization of Lorenz number with Seebeck coefficient measurement, APL materials, 3(4), (2015) 041506.

[14] M. Thesberg, H. Kosina, N. Neophytou, On the Lorenz number of multiband materials, Physical Review B, 95(12), (2017) 125206.

[15] P. J. Price, Theory of transport effects in semiconductors: Thermoelectricity, Physical Review, 104(5), (1956) 1223.

[16] A. Putatunda, D. J. Singh, Lorenz number in relation to estimates based on the Seebeck coefficient, Materials Today Physics, 8, (2009) 49-55.

[17] O. S. Gryazanov, Tables for the calculation of kinetic coefficients in semiconductor, Nauka, Leningrad, 1971.

[18] W. Zawadzki, R. Kowalczyk, J. Kolodziejczak, The Generalized Fermi-Dirac integrals, Physica Status Solidi (b), 10(2), (1965) 513-518.

[19] G. Rzadkowski, S. Łepkowski, A generalization of the Euler-Maclaurin summation formula: an application to numerical computation of the Fermi-Dirac integrals, Journal of Scientific Computing, 35(1), (2008) 63-74.

[20] I. I. Guseinov, B. A. Mamedov, B. A. Unified treatment for accurate and fast evaluation of the FermiDirac functions, Chinese Physics B, 19(5), (2010) 050501.

[21] B. A. Mamedov, E. Copuroglu, Unified analytical treatments of the two-parameter Fermi functions using binomial expansion theorem and incomplete gamma functions, Solid State Communications, 245, (2016) 42-49.

[22] I. M. Ryzhik, I. S. Gradshteyn, Tables of integrals and sums, series, and products, Academic Press, New York, 1980.

[23] E. Çopuroğlu, I. M. Askerov, A. Aslan, Analytical calculation of temperature dependence of the Lorenz number in semiconductors, Journal of Science and Arts, 20(1), (2020)197-202.

[24] M. Shur, Physics of semiconductor devices, Englewood Cliffs, Prentice-Hall, 1990. 